\title{
Analysis of Slow Motion Based on Adaptive Detection Method in Tennis Video
}

\author{
Qiang Zhao ${ }^{1}$ and Yujin Sun ${ }^{2, *}$ \\ ${ }^{1}$ Physical Education Department of Civil Aviation University of China, Tianjin \\ 300300, China \\ ${ }^{2}$ Department of Physical Education, Tianjin University of Science \& Technology, \\ Tianjin 300222, China \\ Itianjinzhaoqiang@sina.cn and ${ }^{2}$ sun@tust.edu.cn \\ 2,*Corresponding author
}

\begin{abstract}
This paper select the tennis video which has logo transition both at the beginning and the end of slow motion as research material. An adaptive threshold based slow motion replay detection method is proposed. This method solves the problem of adaptively selects threshold in the process of slow motion replay detection. Unlike previous approaches, this method has great improvement in both precision rate and positioning accuracy of the boundaries, and can be used for other sports videos which also have logo transition.
\end{abstract}

Keywords: Sports Video, Logo transition, Adaptive threshold

\section{Introduction}

Slow-motion shots are played by studio director to represent highlights in the form of slow motions as to cater audience's psychological needs [1-8]. They contain abundant semantic information. The exploration into slow motion shots has significant meanings: one other hand, it's helpful to create video abstract or generate wonderful collection of a match; on the other hand, the removal of replayed shots is good for professional researchers to analyze better sports video contents or solely make semantic analysis of slow motions [9-16].

In a match, studio director would display spot situations from different angles and aspects, relaying game site in different ways through the whole site, players, court, audience, judges, narrator, normal speed or slow speed [17-20]. Hence according to contents, it can have non-match shots and match shots. Non-match shots refer to those irrelevant with competition like ads, studio; match shots can include slow shots and notslow shots per playback speed (since slow shots are playback of not-slow shots, we intentionally regard it as a type). Not-slow shots are further divided into in-play shots and interval shots; the former refers to game playing; the latter refers to player rest, audience and the full court [21-27].

Since camera can capture views at long or near distance, the shot can be divided into long shot, medium shot and short shot. Long shot generally represents the entire situation of match site; medium shot shows fierce battle during the competition; short shot is closeup of one person, including player, coach, spectators. In-play shot is composed of long shot and medium shot; competition interval shot is consisted of long shot and short shot [28-35]. 


\section{Shot Boundary Detection Technology}

Shot segmentation is an important content of video semantic content analysis, and the accuracy of segmentation directly affects the accuracy of semantic analysis. The premise of the lens segmentation is to detect the shot boundary. The transformation between the lens is mainly divided into two Species: mutation and gradient. A mutation is a direct switch from the current lens to the next; Gradient is defined from the current lens to the next shot transform, there will be a change such as dissolution, wipe, fade out the process [36-37].

The basic idea of the shot boundary detection technology is to compare some features of the adjacent frames. If there is a big change, then the lens transformation occurs, and a larger feature of the place where the camera is located in the boundary. At present, the common shot boundary detection technology mainly has the comparison method based on the pixel, the comparison method based on the block, Based on histogram comparison method, double threshold comparison method, etc [38].

\subsection{Based on the Comparison of Pixel}

Comparison method based on the pixel is on between adjacent frames difference between pixels of summation, then will obtain the difference and same predetermined threshold, if the difference is greater than a threshold, shot change between the two frames, they belong to different lenses. This method is simple in operation, small amount of calculation, but the lens and the moving object is very sensitive.

\subsection{Based on the Block Comparison Method}

Based on block comparison method based on pixel comparison method is similar, but will use the global features of the image change using local features, image is divided into blocks, in the block were compared, the effects it caused to the to the camera and moving object has certain robustness, but at the same time is also much higher than the substrate to like the comparative method of large amount of calculation.

\subsection{Based on the Histogram Comparison Method}

For the effects to further narrow the lens and moving objects on the performance of shot boundary detection, by comparing the histograms of adjacent images. This is because when the background region invariant, object movement caused by the histogram difference is very small and histogram comparison method for image rotation, observation angle and scale change has very good robustness. But this method does not take into account the spatial characteristics of the image, when different frames have the same histogram, the shot boundary cannot be detected.

Using $H_{i}(j)$ to represent the ith frame of the histogram, $\mathrm{n}$ is the interval number of the histogram, $\mathrm{c}$ is the image of the color component, the difference between the $\mathrm{i}$ frame and the $i+1$ frame can be expressed by the following formula:

$$
\begin{aligned}
& D_{i, i+1}=\sum_{j=1}^{n}\left|H_{i}(j)-H_{i+1}(j)\right| \\
& D_{i, i+1}=\sum_{c} \sum_{j=1}^{n}\left|H_{i, c}(j)-H_{i+1, c}(j)\right|
\end{aligned}
$$

In addition, the histogram square difference comparison method can be used, which is the improvement of the histogram comparison method, which can enlarge the distance between adjacent frames: 


$$
D_{i, i+1}=\sum_{i} \frac{\left.H_{i}(j)-H_{i+1}(j)\right)^{2}}{H_{i+1}(j)}
$$

In shot boundary detection, were extracted first the characteristics of two adjacent frames, then according to which a formula of computing the difference between them, if the difference exceeds the preset threshold is considered a shot change between two frames, or continue to turn to the next frame to judge.

\subsection{Double Threshold Comparison Method}

Shot boundary detection technology above to determine the final boundary points are required depends on the threshold and threshold selection is suitable or is not directly affect the accuracy of shot boundary detection. The usual practice is to frame the difference between the frame and the average value plus a small tolerance value as a threshold, which can be used for the detection of abrupt changes in the lens, but the effect is not ideal for gradual change detection.

\section{Adaptive Threshold Detection Method for Slow-Motion Shots}

When watching sports program, we can find in the beginning and ending of playing slow shots, there's shot cut with a Television station LOGO or some sports organization mark appearing in the middle of TV screen, which is called LOGO conversion shot. Since highlights are between two LOGO conversion shots, we can inspect LOGO transformation to realize detection of slow motion shots. Here we explore tennis video slow shots where LOGO conversion exists. For feature extraction, threshold selection and boundary location, we take new method, achieving adaptive selection of threshold during our exploration and obtaining higher boundary location accuracy.

After shot segmentation, a tennis video is cut into numerous shots. Slow motion shot is actually a shot group formed by a set of shots. The LOGO conversion shots in the beginning and ending are separated apart. If we detect the two LOGO conversion shots and make respective boundary frames as beginning and ending of slow shot clip, it's possible to locate roughly the boundary of slow shots. The proposed show shot detection algorithm has following ideas: firstly, make shot segmentation of video streaming to find out candidate LOGO shots from therein; then, generate LOGO template; next make similarity matching of video frame and template in the candidate LOGO shots; according to matching result, determine adaptively threshold value size and locate roughly boundary of slow shots; finally to make detection result more accurate, utilize the change of brightness during LOGO conversion to further locate boundary of slow shots. Algorithm flow chart is shown in Figure 1. 


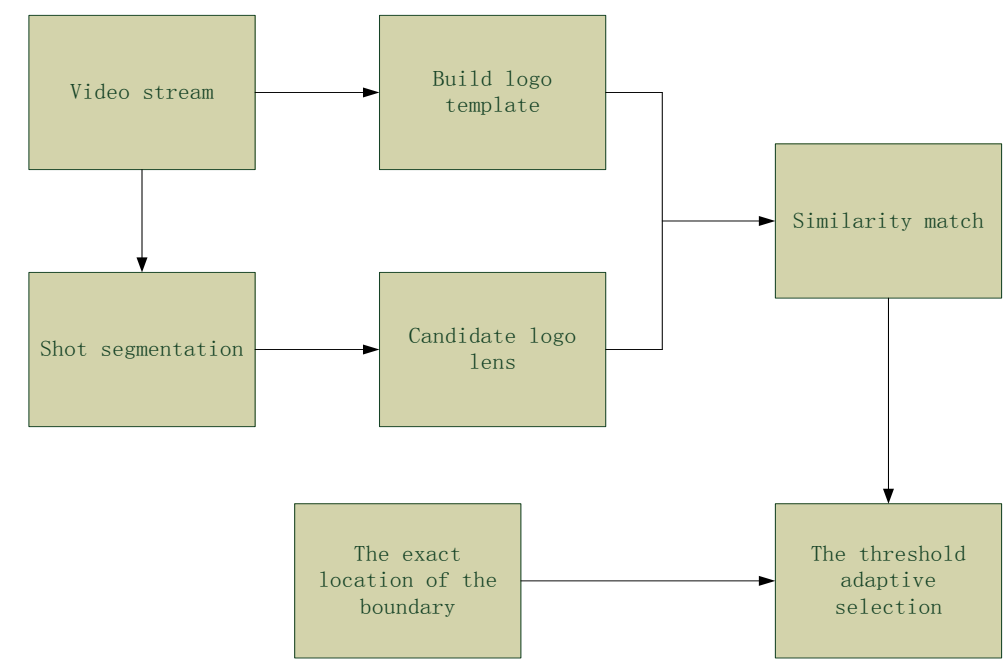

Figure1. Flow Chart of Adaptive Threshold Detection Method for Slow Motion Camera

\subsection{Shot Segmentation Results}

According to the shot boundary detection technology, a double threshold comparison method is used for the shot segmentation.

Figure 2 lists the three shot segmentation results, representing the end of front shot two frames. The start of after shot two frame.
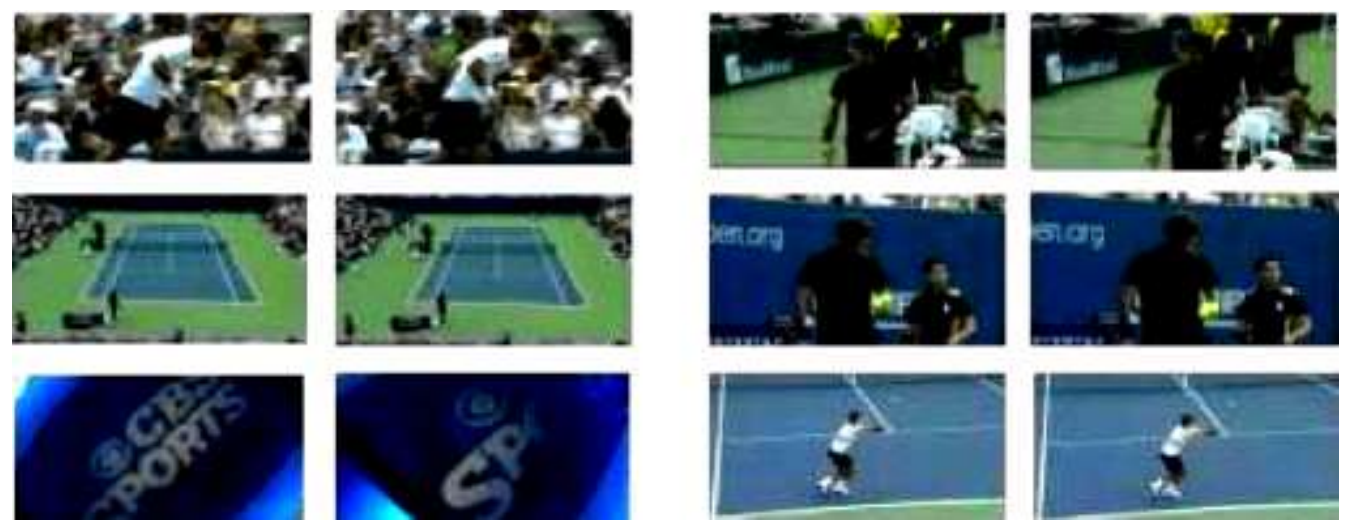

Figure 2. Shot Segmentation Results

\subsection{Selection of Candidate LOGO Shots}

Through observations on lots of tennis videos with LOGO conversion shots, LOGO shots last for 13-22 frames; in that range, shots included are LOGO shots and close-up, of which most are LOGO shots. So we use shots lasting for 13-22 frames after shot segmentation of video streaming as candidate LOGO shot.

\subsection{Extraction of LOGO Template}

LOGO template is extracted usually in three ways: (i) artificial extracting method: pick up the frame with obvious LOGO mark directly from video frame sequence; (ii) semiautomatic extraction method, such as choose by hand several frames which contain obvious LOGO sign [39]; then utilize frame average method to calculate their mean value 
and use as LOGO template; (iii) automatic extraction method: from 3-5 candidate LOGO shots, choose one candidate LOGO frame, then cluster those candidate frames; calcaulate the mean value of candidate LOGO frames around the clustering center and use as LOGO template [40]. In the paper, the extraction of LOGO template is realized by semiautomatic method.

\subsection{Similarity Matching}

\subsubsection{Color Correlogram}

Color correlogram is a way to express image color distribution. The feature not only portrays the proportion of one color's pixel quantity of the whole image and also reflects spatial correlation between different color pairs. It can realize higher retrieval efficiency than color histogram and color coherence vector, especially retrieving images whose spatial relation is consistent.

Suppose I means all pixels of the whole image; ${ }^{I(j)}$ is all pixels of color as $c(i)$. Color correlationgram can be expressed as:

$$
\gamma_{i, j}^{(k)}=\underset{p_{1} \in I_{c(i),}, p_{2} \in I}{P_{1}}\left[p_{2} \in I_{c(j)}\left|p_{1}-p_{2}\right|=k\right]
$$

Color correlogram can be treated as a table indexing by color pair $\langle i, j\rangle$, where the kth component refers to the probability of the distance between pixel of color $c(i)$ and that of color $c(j)$ being less than $\mathrm{k}$. Color correlogram can provide the best retrieving effect; however, if correlation between any colors is considered, color correlogram will get very complicated and huge, spatial complexity up to $O\left(n^{2}\right)$. One simplified variant is color autocorrelogram, which considers only sptial relationship between images having the same color. So, spatial complexity is decreased to $(O(n))$.

\subsubsection{Matching Method}

Since color autocorrelogram has advantages on image retrieval and calculation amount, after LOGO template is got, we employ color autocorrelation characteristics to perform similarity matching of video frame and template in candidate LOGO shots. LOGO pattern often lies in the center of video frames; color autocorrelation characteristics of close-up shot video frame central area are apparently discernible from LOGO shot frames. So by color autocorrelation characteristics, we can separate LOGO shots and close-up. Meanwhile, in order to reduce computation, we segment video frames and LOGO template in candidate LOGO shots both by 1:2:1 in two directions, only analyzing and processing contents in central region. At first, we fetch color autocorrelation information of template and that of each frame in candidate LOGO shots; insert them in MySQL database; then take euclidean distance method to compute respectively the distance between each frame and template; insert calculated distance result into video frame related lines in database table, as to use later for locating boundary of slow shots.

$$
\text { Dis } \tan c e=\sum_{i=1}^{n}\left(\mu_{i}-v_{i}\right)^{2}
$$

\subsection{Adaptive Threshold Selection}

The selection of threshold is a very critical issue. Since video contents differ greatly from each other, a threshold may be effective for one video clip, but not for another video, so it's very difficult to pre-define a suitable threshold value. The threshold here in the proposed slow-motion detection algorithm being too big or small will affect the final 
precision ratio and boundary locating accuracy. To avoid the influence by artificial specified threshold or experical threshold, we proposed a threshold adaptive selection method.

After similarity matching with template through Euclidean distance formula, the distance of LOGO frames is smaller than non-LOGO frames. The objective here is to adaptively choose a threshold and ensure frames to which the distance being less than the threshold corresponds are all LOGO frames. We sort all frames in order by Euclidean distance from small to big after similarity matching. The result is LOGO frames stay ahead and non-LOGO frames stay behind. Finding out a breaking point, we can separate correctly two kinds of frame. The value of distance at the point is threshold value. Considered from the perspective of variance, if other-type frames in one class after classification are fewer, meaning the variance of the class is smaller. If LOGO frames and non-LOGO frames are correctly divided, the variance of two classes is the smallest, thus the variance sum of them is the smallest. The chosen threshold in the paper is the smallest point which makes variance summation the minimum of two classes after classification. The algorithm is carried out in following steps:

(1) Use calcaulted Distance $=\{$ dis1,dis $2, \ldots$ disk $\}$ result by Euclidean distance formula to read from database and sort in ascending order; get the array Dis_sort $=\{\mathrm{D} 1, \mathrm{D} 2, \ldots, \mathrm{DK}\}$ after ranking, where $\mathrm{k}$ is the number of video frames;

(2) Make cyclic variable $\mathrm{i}$ valuing from 1 to $\mathrm{k}$; calculate respectively variance of $\{\mathrm{D} 1, \ldots, \mathrm{Di}\}$ and $\{\mathrm{D}(\mathrm{i}+1), \mathrm{D}(\mathrm{i}+2), \ldots, \mathrm{Dk}\}$ to get summation Ai of them; compute variable $\mathrm{i}$ from 1 to $\mathrm{k}$, one-dimensional variance array $\{\mathrm{A} 1, \mathrm{~A} 2, \ldots, \mathrm{Ak}\}$ is obtained;

(3) Search from one-dimensional variance array $\{\mathrm{A} 1, \mathrm{~A} 2, \ldots, \mathrm{Ak}\}$ the smallest $\mathrm{Ai}$; write down subscript $\mathrm{i}$, corresponding to $\mathrm{Di}$ in the array Dis_sort, which is acquired adaptive threshold value.

The adaptive threshold selection method proposed here is suitable for all sports videos with LOGO conversion, because after template matching, the selection of threshold is not relative with video contents. Instead, dividing all frames into LOGO frames and nonLOGO frames are from the perspective of minimum inter-class variance after correct classification. The flexibility of the algorithm is strengthened, able to optimize by itself along with different video contents.

\subsection{Location of Slow Shot Boundary}

\subsubsection{Initial Location of Slow Shot Boundary}

After the threshold is calculated adaptively, fetch all frames to which the distance in Distance being smaller than Di corresponds; the outcome is many sets of continuous frame collections. Arrange those frame collects as $\left(S_{1}, S_{2}, \ldots, S_{n}\right)$ according to frame order, where $\mathrm{n}$ is number of frame collection. Because slow shots appear between two LOGO conversion shots, for frame collection in odd number of segments, which is believed to be LOGO shot starting from slow-motion shot, we choose from it the most preceding frame as starting point of slow shots; for frame collection in even number of segments, which is believed to be LOGO shot ending in slow-motion shot, we choose from it the most posterior frame as ending point of slow shot; till this moment, boundaries of a segment of slow shot are initially located.

If LOGO conversion shots are missed from detection, there will make mistakes to follow the above method to match LOGO conversion shot as to locate slow shots. Through plenty of experimental observations, we realize slow-motion shot contain the most 10 shots. If the number of shots between two LOGO conversion shots is above 10 , it's thought that missing detection exists in LOGO shots. In this case, we need give up the last one LOGO shot, letting next one LOGO shot matching with its subsequent LOGO shots, which is likely to solve effectively mismatching problem. 


\subsubsection{Precise Positioning of Slow-Motion Shot Boundary}

Since LOGO conversion is a gradual changing process. LOGO sign is not prominent when it appears and disappears. The slow-motion shot boundaries are not often precise if determined with adaptive threshold the method, generally 3-4 frames of LOGO frame can't be detected. However after observations, we see the brightness in those video frames' central region changes too big. Get 5-6 frames ahead / backwards from the starting /ending point of calibrated slow motion; use posterior frame to subtract anterior frame; then extract its brightness value and calculate its cumulative sum. The place where luminance sum is the biggest suggests LOGO begins to appear (about to disappear); choose frame at the place as boundary of slow-motion shot to realize accurate positioning of slow shot boundary. Figure 3 is effect picture of slow shot boundary after precise positioning, where the first line is boundary of anterior LOGO shot; the second line is partial middle slow shot frame; the third line is boundary of posterior LOGO shot.
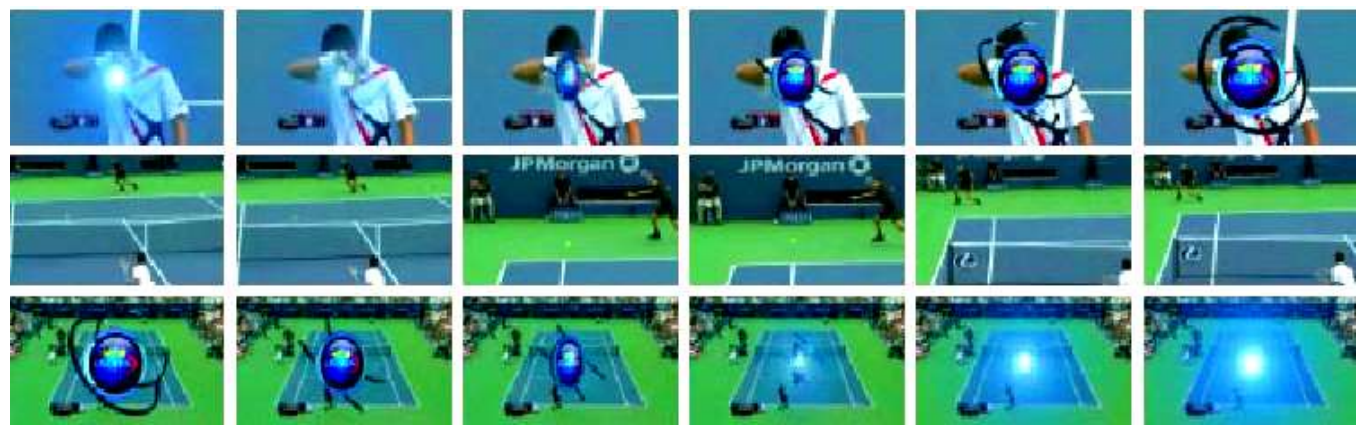

Figure 3. After an Accurate Positioning of the Slow Shot Boundary Effect Chart

\section{Experimental Analysis and Results}

\subsection{Experiment Results}

Two criteria usually used to evaluate goodness or badness of one algorithm's detection effect is recall rate and precision ratio. Here we utilized boundary positioning accuracy rate to measure precision of the algorithm. Of it, missing frame number means those belong to frame containing LOGO but not detected.

$$
\begin{gathered}
\text { Recall }=\frac{\text { Detect the correct number of slow shot }}{\text { Total number of slow shot in video }} \\
\text { Precision }=\frac{\text { Detect the correct number of slow shot }}{\text { Detect the total number of slow shot }} \\
\text { Accuracy of boundary location }=\frac{\text { Number of frames }}{\text { The logo area total frames conversion }}
\end{gathered}
$$

We choose five tennis match videos which contain LOGO transformation as experimental data, validating the proposed algorithm and slow shot detection technique in [17-18]. It is shown in Table1and Table2.

Experiment results reveal that the proposed slow shot detection algorithm can detect well slow motion shots in videos and achieve higher precision rate and boundary positioning accuracy. Recall ratio is affected because errors exist during shot segmentation, causing that a few LOGO shots are not detected when candidate LOGO shots are being detected. The algorithm has better precision ratio and boundary positioning accuracy than existing slow motion shot detection approaches, because the 
utilization of color autocorrelation guarantees image spatial correlation during matching, and adaptive selection of threshold assures that after initial location, what's all acquired are shots containing LOGO conversion, and LOGO shot mismatching problem is corrected, making the algorithm with higher precision ratio. Lastly, on the basis of initially positioning slow shot boundary, the use of brightness changes during LOGO conversion to position boundary accurately increases remarkably the precision of boundary location.

\section{Table 1. Experimental Results of the Slow Shot Detection Algorithm are Presented in this Paper}

\begin{tabular}{|l|l|l|l|l|l|l|}
\hline $\begin{array}{l}\text { Video } \\
\text { sequence } \\
\text { number }\end{array}$ & $\begin{array}{l}\text { Actual } \\
\text { number }\end{array}$ & $\begin{array}{l}\text { Correct } \\
\text { number }\end{array}$ & $\begin{array}{l}\text { Error } \\
\text { number }\end{array}$ & $\begin{array}{l}\text { Recall } \\
(\%)\end{array}$ & $\begin{array}{l}\text { Precision } \\
(\%)\end{array}$ & $\begin{array}{l}\text { Accuracy } \\
\text { of } \\
\text { boundary } \\
\text { location } \\
(\%)\end{array}$ \\
\hline 1 & 18 & 17 & 0 & 93.5 & 100 & 0.4 \\
\hline 2 & 16 & 17 & 0 & 95.8 & 100 & 0.5 \\
\hline 3 & 24 & 22 & 1 & 91.2 & 96.3 & 0.5 \\
\hline 4 & 31 & 27 & 0 & 93.5 & 100 & 0.4 \\
\hline 5 & 35 & 29 & 1 & 93.6 & 96.6 & 0.6 \\
\hline
\end{tabular}

Table 2. Experimental Results of Slow Shot Detection Algorithm in [41-42]

\begin{tabular}{|c|c|c|c|c|c|c|}
\hline \multirow{2}{*}{$\begin{array}{l}\text { Video } \\
\text { sequence } \\
\text { number }\end{array}$} & \multicolumn{3}{|c|}{$\begin{array}{l}\text { Slow shot detection algorithm in } \\
\text { [42] }\end{array}$} & \multicolumn{3}{|c|}{$\begin{array}{l}\text { Slow shot detection algorithm } \\
\text { in [42] }\end{array}$} \\
\hline & $\begin{array}{l}\text { Recall } \\
(\%)\end{array}$ & $\begin{array}{l}\text { Precision } \\
(\%)\end{array}$ & $\begin{array}{l}\text { Accuracy of } \\
\text { boundary } \\
\text { location }(\%)\end{array}$ & $\begin{array}{l}\text { Recall } \\
(\%)\end{array}$ & $\begin{array}{l}\text { Precision } \\
(\%)\end{array}$ & $\begin{array}{l}\text { Accuracy } \\
\text { of } \\
\text { boundary } \\
\text { location } \\
(\%)\end{array}$ \\
\hline 1 & 90 & 94.5 & 2.1 & 90 & 94.3 & 2.5 \\
\hline 2 & 93.9 & 94.5 & 2.3 & 89.6 & 95.7 & 2.3 \\
\hline 3 & 87 & 92.7 & 2.5 & 88 & 91.3 & 3.2 \\
\hline 4 & 92.5 & 90.4 & 2.8 & 89.6 & 92.6 & 2.8 \\
\hline 5 & 93.6 & 90.9 & 2.6 & 90.6 & 95.6 & 2.9 \\
\hline
\end{tabular}

\subsection{Algorithm Complexity Analysis}

The adaptive threshold detection method introduced here for slow-motion shots in tennis videos includes shot segmentation, candidate LOGO shot selection, LOGO template extraction, similarity matching, adaptive threshold selection and slow-motion boundary positioning. Of them, the core is adaptive threshold selection, because the choice of threshold matters directly the accuracy of algorithm detecting slow motion shots. For the selection of threshold, we used one-dimension array as sample space, getting step by step the variance of each component array and sum them up; then seek for the smallest variance sum; the time complexity is $O(n)$ and spatial complexity is likewise $O(n)$. Besides, in other steps of the algorithm, the biggest complexity of time and space is $O(n)$. Accordingly, the time and space complexity of slow-motion shot detection algorithm here is both $O(n)$. 


\section{Conclusion}

In this paper, we firstly introduce the shot in tennis video shot boundary detection and classification of related knowledge; Secondly according to the algorithm process is introduced in detail the slow motion detection method; in the end of this paper, put forward the method of the experiment results and algorithm complexity analysis, and comparing the results with the other two methods. Experimental results show that this method has better detection results.

\section{References}

[1] Z. Xiaoli and C. Min, "An adaptive threshold detection method for slow motion in tennis video", Computer engineering and science, vol. 4, (2013), pp. 99-103.

[2] L. Dian, "Soccer video slow motion detection and video summary generation", North China University of Technology, (2008).

[3] L. Kun, "Research of soccer video retrieval based on MPEG", Jilin University, (2007).

[4] Z. Jujube, "Research and implementation of the slow motion detection technology in sports video", University of national defense science and technology, (2004).

[5] G. Shuwen, "Classification and retrieval methods of soccer videos", Huazhong University of Science and Technology, (2012).

[6] L. Huifang, "Multi level analysis and retrieval of tennis video", Nanjing University of Science and Technology, (2009).

[7] W. Haibo, "Research on the detection technology of sports video events", North China Electric Power University (Beijing), (2009).

[8] P. Ruifang and Z. Ming, "Research on the application techniques of long shot in documentary film", Press, vol. 1, (2012), pp. 24-27.

[9] L. Bin, L. Qun, W. Qun and N. Yanliu, "Classification method of soccer video camera", Computer engineering and design, vol. 4, (2012), pp. 1467-1471.

[10] Z. Yuzhen, D. Sijie, W. Jianyu, D. Yuewei and C. Qian, "Multi-modality event detection based on HMM fusion", Journal of system simulation, vol. 8, (2012), pp. 1638-1642.

[11] W. Haoran, B. Liang and Y. S. Yang, "Semantic modeling method for soccer video based on graph model”, Computer science, vol. 6, (2011), pp. 266-269.

[12] L. Haitao, S. Yang and B. Jiang, "Video semantic content analysis based on ontology", Computer science, vol. 7, (2009), pp. 170-174.

[13] Y. Junqing and W. Ning, "Classification of soccer video shots based on sub window region", Chinese Journal of image and graphics, vol. 7, (2008), pp. 1347-1352.

[14] Z. Panhua, R. Keqiang and X. Bin, "An adaptive learning algorithm for moving object detection based on the mixed Gauss model", Computer engineering and design, vol. 3, (2014), pp. 968-974.

[15] L. Jianwei, S. Zhengkang and L. Xionglin, "Domain adaptive learning progress", Journal of automation, vol. 8, (2014), pp. 1576-1600.

[16] L. Xucheng, Z. Xiao and L. Mingyu, "Based on Hamiltonian Markov chain Monte Carlo method of abrupt motion tracking", Journal of software, vol. 7, (2014), pp. 1593-1605.

[17] H. Q. Min, L. Jumei, L. R. Hua and C. Cong, "Based on GMM and MRF adaptive shadows detection", South China University of Technology (NATURAL SCIENCE EDITION) Journal, vol. 7, (2011), pp. 115-120.

[18] Y. Junchao and L. Zhifang, "Detection and tracking of moving objects", OpenCV computer engineering and design, vol. 8, (2011), pp. 2817-2820.

[19] L. Gang, H. Xiaohai, Z. Shengjun and G. Bright, "An improved GMM based moving object detection method", Computer application research, vol. 12, (2011), pp. 4738-4741

[20] L. Hong, S. Yigang and Z. Hongying, "A moving object detection algorithm based on improved mixed Gauss model”, Computer Engineering, vol. 18, (2012), pp. 166-170.

[21] W. Ling and L. Jing, "An improved algorithm for the background of mixed Gauss model", Computer engineering and application, vol. 13, (2010), pp. 168-170.

[22] Z. Yuzhen, W. Daidi, W. Jianyu and D. Yuewei, "Semantic analysis of soccer video based on multimodal fusion", Computer science, vol. 7, (2010), pp. 273-276.

[23] L. Xiang, G. Guangzhu and H. Zhiyong, "Research on intelligent detection algorithm for moving objects", modern electronic technology, vol. 19, (2010), pp. 76-80.

[24] G. P. Zhao and T. Yuming, "Based on spatiotemporal multimodal mean model of target fusion detection method", Journal of image and graphics, vol. 8, (2010), pp. 1254-1259.

[25] L. Jianwu, Z. Yingying and S. Na, "The main color clustering algorithm of game field with fusion audio features", Journal of Putian University, vol. 5, (2010), pp. 55-59.

[26] X. T. Zheng and IBM, "A based on improved codebook model fast motion detection algorithm", Journal of computer research and development, vol. 12, (2010), pp. 2149-2156. 
[27] J. Xiaolian, L. Cuihua, L. Kai and L. Wei, "A Wang-Landau Monte Carlo sampling mutation target tracking algorithm based on visual saliency", Journal of Xiamen University (NATURAL SCIENCE EDITION), (2013,04:498-505.

[28] Y. Ningxue and L. Yanping, "Human motion analysis and its application in 3D interactive entertainment", Electronic test, vol. 10, (2013), pp. 122-125.

[29] D. X. Qiang and Z. Dake, "Deer music. Matching based on vehicle detection algorithm based on distribution and Gaussian mixture model", Jilin University Journal of information science and, vol. 5, (2013), pp. 540-547.

[30] Z. Wangfei, S. X. An and H. Y. Qiu, "Soup Shusen. Improved based on Gaussian mixture model of the method of moving target detection", Computer application, vol. 10, (2007), pp. 2544-2546

[31] Y. Junqing, L. Chong, H. Yunfeng and H. Shenghong, "Adaptive transmission video content using motion intensity", Computer aided design and computer graphics, vol. 6, (2009), pp. 847-852.

[32] Y. Maoyong, J. Yang and Z. Rui, "Hong sentinel. Based on analysis of the background Gaussian mixture background model establishment", Security technology, vol. 4, (2009), pp. 3-7.

[33] L. Ling, "A background adaptive moving target detection algorithm”, Journal of Shaanxi University of Technology (NATURAL SCIENCE EDITION), vol. 6, (2014), pp. 40-45.

[34] S. Haifeng, L. Wei and C. Chung, "Static background extraction and update algorithm of adaptive weights", Journal of electronic measurement and instrument, vol. 3, (2015), pp. 358-367.

[35] Z. Jin and L. Bo, "Video moving object detection based on three order center moment region classification", Journal of computer aided design and computer graphics, vol. 5, (2015), pp. 832$840+846$.

[36] Y. Yao, S. Songzhi, W. Li and L. Shaozi, "Detection method of moving objects based on improved mixed Gauss model", Journal of Xiamen University (NATURAL SCIENCE EDITION), vol. 4, (2008), pp. 505-510.

[37] L. Ya, A. Haizhou and X. Guangyou, "An algorithm for moving object detection and tracking based on background model", information and control, vol. 4, (2002), pp. 315-319

[38] H. J. Zhang, A. Kankanhalli and S. W. Smoliar, "Automatic partitioning of full motion video", Multimedia System, vol. 1, no. 1, (1993), pp. 10-28

[39] X. Tong, H. Lu, Q. Liu and H. Jin, "Replay Detection in Broadcasting Sports Video", In: International Conference on Image and Graphics(ICIG), (2004), pp. 337-340.

[40] L. Xiaowei, "Sports video analysis and personalized customization", Wuhan: Wuhan University of Technology, (2010).

[41] Z. Jujube, "Research and implementation of the slow motion detection technology in sports video", Changsha: National University of Defense Science and technology, (2004).

[42] Z. Yuzhen, W. Daidi, W. Jianyu and D. Yuewei, "Semantic analysis of soccer video based on multimodal fusion", Computer science, vol. 37, no. 7, (2010), pp. 273-276.

\section{Authors}

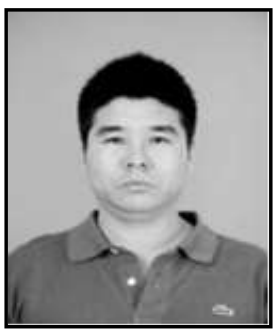

Qiang Zhao, He received his B.S and M.S degree from Tianjin University of Sport. He is an associate professor at Physical Education Department of Civil Aviation University of China. He is in the research of Physical Education and Training. 